\title{
ANALYSIS OF DISCRETE-TIME H2 GUARANTEED COST PERFORMANCE
}

\author{
Richard Conway \\ Computer Mechanics Laboratory \\ Department of Mechanical Engineering \\ University of California at Berkeley \\ Berkeley, California 94720-1740 \\ Email: rconway345@berkeley.edu
}

\author{
Roberto Horowitz \\ Professor of Mechanical Engineering \\ University of California at Berkeley \\ Berkeley, California 94720-1740 \\ Email: horowitz@me.berkeley.edu
}

\begin{abstract}
This paper presents a methodology for analyzing the $\mathcal{H}_{2}$ guaranteed cost performance of a discrete-time LTI system with unstructured dynamic uncertainty. Using the methods of guaranteed cost control, an upper bound on $\mathcal{H}_{2}$ guaranteed cost performance over unstructured parametric uncertainty is formulated in terms of feasibility of a linear matrix inequality. It is then shown that the feasibility of this inequality also guarantees the same level of performance also over unstructured dynamic uncertainty. This is then used to formulate the problem of finding the best upper bound on $\mathcal{H}_{2}$ guaranteed cost performance over unstructured causal dynamic uncertainty as a semi-definite program. Finally, it is shown that this optimization problem can be solved efficiently and accurately using discrete algebraic Riccati equations.
\end{abstract}

\section{Introduction}

The $\mathcal{H}_{2}$ norm has long been the most widely-used measure of performance for stable discrete-time LTI systems. There are two reasons why this is the case. From a computational standpoint, the $\mathcal{H}_{2}$ norm is easy to calculate because it only requires the solution of a single Lyapunov equation and standard matrix manipulations. From an intuitive standpoint, the squared $\mathcal{H}_{2}$ norm of an LTI system can be interpreted as the trace of the steady-state system output covariance under the assumption that the system is driven by white Gaussian noise with unit covariance. Since many disturbances of interest can be modeled as Gaussian noise (either white or filtered), this makes the $\mathcal{H}_{2}$ norm a particularly useful measure of performance when the system and its disturbances are well-characterized.

However, it is often the case that the system and/or its dis- turbances are not well-characterized. In this case, it is customary to model the uncertainty in the system model and express the resulting model as a linear fractional transformation (LFT) of a known state space system and an unknown transfer function with a $\mathcal{H}_{\infty}$ norm bound which represents the uncertainty in the model.

In this framework, we are interested in determining the worst-case $\mathcal{H}_{2}$ performance of the discrete-time system over all modeled uncertainty. In general, the unknown part of the system could have some structure, such as in $\mu$-synthesis. Necessary and sufficient conditions for robust $\mathcal{H}_{2}$ performance in this case are derived in the frequency domain in [1]. The resulting conditions need to be checked at every frequency (or at least a fine grid of frequencies) and then integrated across frequency. In that paper, these conditions are then extended to state space systems and the resulting optimization problem is reduced to a convex optimization problem involving a finite number of linear matrix inequalities (LMIs). However, in both of these approaches, there is a significant amount of conservatism that arises because they do not make any assumptions on the causality of the unknown part of the system.

A related approach for guaranteeing robust performance of a system over model uncertainty is guaranteed cost control [2]. The analysis results of this framework are different than the previous framework in two ways. First, they are time domain analysis results instead of a frequency domain results. Second, the analysis only applies to systems with parametric uncertainty.

This paper uses the techniques of guaranteed cost control to derive a condition which yields an upper bound on robust $\mathcal{H}_{2}$ performance of a discrete-time LTI system over unstructured normbounded parametric uncertainty. It is then shown that the same condition also guarantees robust $\mathcal{H}_{2}$ performance over causal unstructured dynamic uncertainty with the same $\mathcal{H}_{\infty}$ norm bound. 
The problem of finding the best $\mathcal{H}_{2}$ guaranteed cost performance is then formulated as a semi-definite program (SDP), which can be solved using solvers such as SeDuMi [3] or by using the mincx command in the Robust Control Toolbox for MATLAB. An efficient algorithm for solving this convex optimization problem is then developed which exploits the structure of the problem by using the solutions of discrete algebraic Riccati equations (DAREs). This algorithm is analogous to the algorithm developed for continuous-time systems in [4]. It is then shown that the resulting algorithm is faster and tends to be more accurate than using general convex optimization routines to solve the SDP.

Throughout the paper, we will use the following notation and definitions. A matrix will be called Schur if all of its eigenvalues lie strictly inside the unit disk in the complex plane. A matrix pair $(A, B)$ will be called d-stabilizable if $\exists K$ such that $A+B K$ is Schur. Positive definiteness (semi-definiteness) of a symmetric matrix $X$ will be denoted by $X \succ 0(X \succeq 0)$, bullets in a matrix will represent elements of a matrix which follow from symmetry, and the maximum singular value of a matrix $M$ will be denoted $\|M\|$. Finally, the $\mathcal{H}_{2}$ norm and $\mathcal{H}_{\infty}$ norm of a stable causal LTI system $G(z)$ will be denoted respectively by $\|G(z)\|_{2}$ and $\|G(z)\|_{\infty}$.

\section{RICCATI EQUATIONS AND LINEAR MATRIX IN- EQUALITIES}

\subsection{DISCRETE ALGEBRAIC RICCATI EQUATIONS}

We start by defining

$$
\begin{aligned}
\phi:= & (A, B, Q, R, S) \\
\mathcal{R}_{\phi}(P):= & A^{T} P A+Q-P \\
& -\left(A^{T} P B+S\right)\left(B^{T} P B+R\right)^{-1}\left(B^{T} P A+S^{T}\right) \\
\mathcal{K}_{\phi}(P):= & -\left(B^{T} P B+R\right)^{-1}\left(B^{T} P A+S^{T}\right) \\
\mathcal{A}_{\phi}(P):= & A+B \mathcal{K}(P) .
\end{aligned}
$$

for given values of $A, B, Q, R$, and $S$ where $Q$ and $R$ are symmetric and $A$ is square. These quantities will play a pivotal role in the developments of this section and section 4 . For a given value of $\phi$, the equation $\mathcal{R}_{\phi}(P)=0$ is a DARE. We will say that $P$ is a stabilizing solution of the DARE if $\mathcal{R}_{\phi}(P)=0, P=P^{T}$, and $\mathcal{A}_{\phi}(P)$ is Schur. We will say that $P$ is a maximal solution of the DARE if $\mathcal{R}_{\phi}(P)=0$ and $P \succeq \bar{P}$ for any other DARE solution, $\bar{P}$.

In this section, we review two standard results on DAREs. Although, for brevity, we do not prove either of these results, both proofs can be constructed using only discrete Lyapunov equations, Sylvester equations, and elementary matrix manipulations. The following proposition is a trivial corollary of Proposition 13.5.1 in [5].

Proposition 2.1. If a DARE has a stabilizing solution, then it is unique.
The following proposition, which is a trivial corollary of Theorem 13.1.3 in [5], is a standard result in the design of LQR controllers and Kalman filters.

Proposition 2.2. If $(A, B)$ is d-stabilizable and $\left[\begin{array}{cc}Q & S \\ S^{T} & R\end{array}\right] \succ 0$ then the DARE $\mathcal{R}_{\phi}(P)=0$ has a maximal stabilizing solution $P_{0} \succ 0$.

\subsection{MINIMAL LMI SOLUTIONS VIA DARES}

We begin by defining $\mathcal{L}_{\phi}$ as

$$
\mathcal{L}_{\phi}(P):=\left[\begin{array}{cc}
A^{T} P A+Q-P & A^{T} P B+S \\
\bullet & B^{T} P B+R
\end{array}\right]
$$

with the vector space of symmetric matrices as its domain. Note that because $\mathcal{L}_{\phi}(P)-\mathcal{L}_{\phi}(0)$ is a linear function of $P$, constraining $\mathcal{L}_{\phi}(P)$ to be positive or negative definite or semi-definite is an LMI. We now establish necessary and sufficient conditions for the feasibility of the LMI $\mathcal{L}_{\phi}(P) \succ 0$ under the condition that $(A, B)$ is d-stabilizable.

Lemma 2.3. Let $(A, B)$ be d-stabilizable. The DARE $\mathcal{R}_{\phi}(P)=0$ has a stabilizing solution $P_{0}$ such that $B^{T} P_{0} B+R \succ 0 \Leftrightarrow \exists P$ such that $\mathcal{L}_{\phi}(P) \succ 0$. Moreover, $\mathcal{L}_{\phi}(P) \succ 0 \Rightarrow P_{0} \succ P$.

Proof. $(\Rightarrow)$ Let $P_{0}$ be the stabilizing solution of the DARE satisfying $B^{T} P_{0} B+R \succ 0$. This implies that $\mathcal{A}_{\phi}\left(P_{0}\right)$ is Schur. Now we choose a Lyapunov function for $\mathcal{A}_{\phi}\left(P_{0}\right)$, i.e. let $X \succ 0$ satisfy $X-\mathcal{A}_{\phi}\left(P_{0}\right)^{T} X \mathcal{A}\left(P_{0}\right) \succ 0$ and then define $Y(t):=P_{0}-t X$. Note that $\mathcal{R}_{\phi}(Y(0))=0$ and, after some algebra,

$$
\left.\frac{d \mathcal{R}_{\phi}(Y(t))}{d t}\right|_{t=0}=X-\mathcal{A}_{\phi}\left(P_{0}\right)^{T} X \mathcal{A}_{\phi}\left(P_{0}\right) \succ 0 .
$$

Thus, for sufficiently small $t>0$, we see that $\mathcal{R}_{\phi}(Y(t)) \succ 0$ and $B^{T} Y(t) B+R=\left(B^{T} P_{0} B+R\right)-t B^{T} X B \succ 0$. By Schur complements, these two conditions are equivalent to $\mathcal{L}_{\phi}(Y(t)) \succ 0$.

$(\Leftarrow)$ Choose any $\Delta=\Delta^{T}$ such that $\mathcal{L}_{\phi}(\Delta) \succ 0$ and define $\bar{Q}$, $\bar{R}$, and $\bar{S}$ as

$$
\left[\begin{array}{cc}
\bar{Q} & \bar{S} \\
\bar{S}^{T} & \bar{R}
\end{array}\right]:=\left[\begin{array}{cc}
A^{T} \Delta A+Q-\Delta & A^{T} \Delta B+S \\
\bullet & B^{T} \Delta B+R
\end{array}\right]=\mathcal{L}_{\phi}(\Delta) \succ 0 .
$$

Also define $\bar{\phi}:=(A, B, \bar{Q}, \bar{R}, \bar{S})$. By Proposition 2.2 , the DARE $\mathcal{R}_{\bar{\phi}}(\bar{P})=0$ has a stabilizing solution $\bar{P}_{0} \succ 0$. Defining $P_{0}:=\bar{P}_{0}+$ $\Delta$, we see that $B^{T} P_{0} B+R=B^{T} \bar{P}_{0} B+\bar{R} \succ 0$. Similarly, $\mathcal{R}_{\phi}\left(P_{0}\right)=$ $\mathcal{R}_{\bar{\phi}}\left(\bar{P}_{0}\right)=0$ and $\mathcal{A}_{\phi}\left(P_{0}\right)=\mathcal{A}_{\bar{\phi}}\left(\bar{P}_{0}\right)$. This implies that $P_{0}$ is the stabilizing solution of the DARE $\mathcal{R}_{\phi}(P)=0$. Since $P_{0}-\Delta=$ $\bar{P}_{0} \succ 0$, we see that $P_{0} \succ \Delta$. Since the choice of $\Delta$ was arbitrary, this concludes the proof. 
With this in place, we can now establish necessary and sufficient conditions for the feasibility of the LMI $\mathcal{L}(P) \prec 0$ under the condition that $(A, B)$ is d-stabilizable.

Theorem 2.4. Let $(A, B)$ be d-stabilizable. The DARE $\mathcal{R}_{\phi}(P)=$ 0 has a stabilizing solution $P_{0}$ such that $B^{T} P_{0} B+R \prec 0 \Leftrightarrow \exists P$ such that $\mathcal{L}_{\phi}(P) \prec 0$. Moreover, $\mathcal{L}_{\phi}(P) \prec 0 \Rightarrow P_{0} \prec P$.

Proof. Defining $\bar{\phi}:=(A, B,-Q,-R,-S)$ we see that $\mathcal{L}_{\bar{\phi}}(-P)=$ $-\mathcal{L}_{\phi}(P)$. Thus, $\exists P$ such that $\mathcal{L}_{\phi}(P) \prec 0$ if and only if $\exists \bar{P}$ such that $\mathcal{L}_{\bar{\phi}}(\bar{P}) \succ 0$. By Lemma 2.3, this is equivalent to the DARE $\mathcal{R}_{\bar{\phi}}(\bar{P})=0$ having a stabilizing solution $\bar{P}_{0}$ such that $B^{T} \bar{P}_{0} B+\bar{R} \succ$ 0 . Defining $P_{0}:=-\bar{P}_{0}$, we see after a little algebra that $\mathcal{R}_{\phi}\left(P_{0}\right)=$ $-\mathcal{R}_{\bar{\phi}}\left(\bar{P}_{0}\right)=0, \mathcal{A}_{\phi}\left(P_{0}\right)=\mathcal{A}_{\bar{\phi}}\left(\bar{P}_{0}\right)$, and $B^{T} P_{0} B+R=-\left(B^{T} \bar{P}_{0} B+\right.$ $\bar{R}) \prec 0$ which establishes the required equivalency.

Now let $\mathcal{L}_{\phi}(P) \prec 0$. This implies that $\mathcal{L}_{\bar{\phi}}(-P)=-\mathcal{L}_{\phi}(P) \succ$ 0 , which in turn implies that $-P \prec \bar{P}_{0}$ by Lemma 2.3. Therefore, $P \succ P_{0}$.

Remark 2.5. Since the stabilizing solution of a DARE is unique, Theorem 2.4 yields a test for checking whether or not the LMI $\mathcal{L}_{\phi}(P) \prec 0$ is feasible. First, try to find the stabilizing solution of the DARE $\mathcal{R}_{\phi}(P)=0$ using a software package such as MATLAB. The LMI is feasible if and only if the stabilizing solution $P_{0}$ exists and satisfies $B^{T} P_{0} B+R \prec 0$.

The previous theorem established equivalence of a stabilizing DARE solution and feasibility of the LMI $\mathcal{L}_{\phi}(P) \prec 0$. The following corollary establishes that this DARE solution is the minimal solution of the nonstrict LMI $\mathcal{L}_{\phi}(P) \preceq 0$.

Corollary 2.6. Let $(A, B)$ be d-stabilizable and let the DARE $\mathcal{R}_{\phi}(P)=0$ have a stabilizing solution $P_{0}$ such that $B^{T} P_{0} B+R \prec$ 0 . Then $P_{0}$ is the minimal solution of the $L M I \mathcal{L}_{\phi}(P) \preceq 0$.

Proof. Using Schur complements, it is easily verified that $P_{0}$ is a solution of the LMI, i.e. $\mathcal{L}_{\phi}\left(P_{0}\right) \preceq 0$. Let $\mathcal{L}_{\phi}(P) \preceq 0$. By Theorem 2.4, we now choose $\Delta=\Delta^{T}$ such that $\mathcal{L}_{\phi}(\Delta) \prec 0$. Now define $Y(\lambda):=\lambda P+(1-\lambda) \Delta$. Note that

$$
\mathcal{L}_{\phi}(Y(\lambda))=\lambda \mathcal{L}_{\phi}(P)+(1-\lambda) \mathcal{L}_{\phi}(\Delta) \prec 0, \quad \forall \lambda \in[0,1) .
$$

Using Theorem 2.4 again, we see that $P_{0} \prec Y(\lambda), \forall \lambda \in[0,1)$. The continuity of $Y(\lambda)$ implies that $P_{0} \preceq Y(1)=P$.

\section{LMI APPROACH TO H2 GUARANTEED COST ANAL- YSIS}

In this section, we use the techniques of guaranteed cost control to analyze the robust $\mathcal{H}_{2}$ performance of a discrete- time LTI system with unstructured parametric uncertainty. We then show that the resulting optimization problem of finding the smallest bound on the $\mathcal{H}_{2}$ guaranteed cost can be formulated as an SDP.

We begin with a proposition that will facilitate later proofs.
Proposition 3.1. $\exists W, V$ such that $\left[\begin{array}{cc}M_{11} & M_{12}(V) \\ \bullet & W-M_{22}\end{array}\right] \succ 0$ if and only if $M_{11} \succ 0$.

Proof. $(\Rightarrow)$ Trivial.

$(\Leftarrow)$ Let $M_{11} \succ 0$ and choose any value of $V$. By Schur complements, $W=I+M_{22}+M_{12}(V)^{T} M_{11}^{-1} M_{12}(V)$ satisfies the matrix inequality.

We now consider a given discrete-time LTI system $G(z)$ with the realization

$$
G(z) \sim\left[\begin{array}{l|l}
\bar{A} & \bar{B} \\
\bar{C} & \bar{D}
\end{array}\right]
$$

The following lemma establishes sharp upper bounds on the $\mathcal{H}_{2}$ norm of $G(z)$ under the assumption that its state space realization is known.

Lemma 3.2. The following are equivalent:

1. $\|G(z)\|_{2}^{2}<\gamma$.

2. $\exists P \succ 0, W$ such that $\gamma>\operatorname{tr}\{W\}, W-\bar{B}^{T} P \bar{B}-\bar{D}^{T} \bar{D} \succ 0$, and $P-\bar{A}^{T} P \bar{A}-\bar{C}^{T} \bar{C} \succ 0$.

3. $\exists P \succ 0, W, V$ such that $\gamma>\operatorname{tr}\{W\}$ and

$$
\left[\begin{array}{cc}
P & V \\
V^{T} & W
\end{array}\right]-\left[\begin{array}{ll}
\bar{A} & \bar{B} \\
\bar{C} & \bar{D}
\end{array}\right]^{T}\left[\begin{array}{ll}
P & 0 \\
0 & I
\end{array}\right]\left[\begin{array}{ll}
\bar{A} & \bar{B} \\
\bar{C} & \bar{D}
\end{array}\right] \succ 0
$$

Proof. Since the LMI characterization of the $\mathcal{H}_{2}$ norm is now considered a standard result in the literature, we only prove the equivalence of the final two conditions. Note that

$$
\begin{aligned}
{\left[\begin{array}{cc}
P & V \\
V^{T} & W
\end{array}\right]-\left[\begin{array}{ll}
\bar{A} & \bar{B} \\
\bar{C} & \bar{D}
\end{array}\right]^{T}\left[\begin{array}{ll}
P & 0 \\
0 & I
\end{array}\right]\left[\begin{array}{ll}
\bar{A} & \bar{B} \\
\bar{C} & \bar{D}
\end{array}\right] } \\
=\left[\begin{array}{cc}
P-\bar{A}^{T} P \bar{A}-\bar{C}^{T} \bar{C} & V-\bar{A}^{T} P \bar{B}-\bar{C}^{T} \bar{D} \\
\bullet & W-\bar{B}^{T} P \bar{B}-\bar{D}^{T} \bar{D}
\end{array}\right] .
\end{aligned}
$$

Thus, condition 3 trivially implies condition 2. Conversely, if condition 2 is satisfied, we can always choose $V=\bar{A}^{T} P \bar{B}+\bar{C}^{T} \bar{D}$ to satisfy condition 3 .

The previous lemma gave a sharp upper bound on the $\mathcal{H}_{2}$ performance of a known system. We now remove the restriction that $G(z)$ is known. In particular, we let $G(z)$ be represented as an LFT of a known state space system and an unknown LTI system $\Delta(z)$ as shown in Fig. 1. The system is thus governed by the equations

$$
\left[\begin{array}{c}
x_{k+1} \\
\hline q_{k} \\
p_{k}
\end{array}\right]=\left[\begin{array}{c|cc}
A & B_{1} & B_{2} \\
\hline C_{1} & D_{11} & D_{12} \\
C_{2} & D_{21} & D_{22}
\end{array}\right]\left[\begin{array}{l}
x_{k} \\
\hline d_{k} \\
w_{k}
\end{array}\right], \quad d(z)=\Delta(z) q(z)
$$




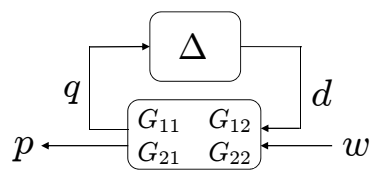

Figure 1. LFT REPRESENTATION OF UNCERTAIN SYSTEM

where $x_{k} \in \mathbb{R}^{n_{x}}, q_{k} \in \mathbb{R}^{n_{q}}, p_{k} \in \mathbb{R}^{n_{p}}, d_{k} \in \mathbb{R}^{d_{x}}$, and $w_{k} \in \mathbb{R}^{n_{w}}$. We now define the static and dynamic uncertainty sets respectively as

$$
\begin{gathered}
\underline{\Delta}_{s}:=\left\{\Delta \in \mathbb{R}^{n_{d} \times n_{q}}:\|\Delta\| \leq 1\right\} \\
\underline{\Delta}_{d}:=\left\{\Delta(z) \in \mathcal{R} \mathcal{H}_{\infty}^{n_{d} \times n_{q}}:\|\Delta(z)\|_{\infty} \leq 1\right\}
\end{gathered}
$$

where $\mathcal{R} \mathcal{H}_{\infty}$ is the set of scalar real proper transfer functions with no poles outside the unit circle. We will regard the elements of $\underline{\Delta}_{d}$ as causal systems. Since the system is robustly well-posed over $\Delta \in \Delta_{s}$ if and only if $\left\|D_{11}\right\|<1$, we will make that assumption throughout the remainder of this section.

We now examine the system under the restriction that $\Delta \in$ $\underline{\Delta}_{s}$. In this case, $G(z)$ has the realization

$$
\begin{gathered}
G(z) \sim\left[\begin{array}{c|c}
A+B_{1} \Phi_{1, \Delta} & B_{2}+B_{1} \Phi_{2, \Delta} \\
\hline C_{2}+D_{21} \Phi_{1, \Delta} & D_{22}+D_{21} \Phi_{2, \Delta}
\end{array}\right]=:\left[\begin{array}{c|c}
A_{\Delta} \mid B_{\Delta} \\
\hline C_{\Delta} \mid D_{\Delta}
\end{array}\right] \\
\Phi_{1, \Delta}:=\left(I-\Delta D_{11}\right)^{-1} \Delta C_{1}, \quad \Phi_{2, \Delta}:=\left(I-\Delta D_{11}\right)^{-1} \Delta D_{12} .
\end{gathered}
$$

For convenience, we define the quantity

$$
\begin{aligned}
\mathcal{M}(\tau, P, W, V) & = \\
& {\left[\begin{array}{ccc}
P & 0 & V \\
\bullet & \tau I & 0 \\
\bullet & \bullet & W
\end{array}\right]-\left[\begin{array}{ccc}
A & B_{1} & B_{2} \\
C_{1} & D_{11} & D_{12} \\
C_{2} & D_{21} & D_{22}
\end{array}\right]^{T}\left[\begin{array}{ccc}
P & 0 & 0 \\
0 & \tau I & 0 \\
0 & 0 & I
\end{array}\right]\left[\begin{array}{ccc}
A & B_{1} & B_{2} \\
C_{1} & D_{11} & D_{12} \\
C_{2} & D_{21} & D_{22}
\end{array}\right] . }
\end{aligned}
$$

This quantity will play a pivotal role in the remainder of this section and the next section. Using the standard techniques for guaranteed cost control [2], the following theorem gives an upper bound for $\|G(z)\|_{2}^{2}$ under the assumption that $\Delta \in \underline{\Delta}_{s}$.

Theorem 3.3. The condition

$$
\left[\begin{array}{cc}
P & V \\
V^{T} & W
\end{array}\right]-\left[\begin{array}{cc}
A_{\Delta} & B_{\Delta} \\
C_{\Delta} & D_{\Delta}
\end{array}\right]^{T}\left[\begin{array}{ll}
P & 0 \\
0 & I
\end{array}\right]\left[\begin{array}{ll}
A_{\Delta} & B_{\Delta} \\
C_{\Delta} & D_{\Delta}
\end{array}\right] \succ 0, \quad \forall \Delta \in \underline{\Delta}_{s}
$$

holds if and only if $\exists \tau>0$ such that $\mathcal{M}(\tau, P, W, V) \succ 0$. Moreover, if $P \succ 0$ and these conditions hold, then $\|G(z)\|_{2}^{2}<\operatorname{tr}\{W\} \forall \Delta \in$ $\Delta_{s}$.

Proof. First note that if (1) holds, it will still hold if we replace $W$ by $W-\varepsilon I$ for small enough $\varepsilon>0$. Thus, if $P \succ 0$, we define $\gamma:=$
$\operatorname{tr}\{W\}>\operatorname{tr}\{W-\varepsilon I\}$ and conclude by Lemma 3.2 that $\|G(z)\|_{2}^{2}<$ $\operatorname{tr}\{W\}, \forall \Delta \in \underline{\Delta}_{s}$.

To prove the equivalence of the required statements, we first define for convenience

$$
L:=\left[\begin{array}{ccc}
P & 0 & V \\
0 & 0 & 0 \\
V^{T} & 0 & W
\end{array}\right]-\left[\begin{array}{ccc}
A & B_{1} & B_{2} \\
C_{2} & D_{21} & D_{22}
\end{array}\right]^{T}\left[\begin{array}{cc}
P & 0 \\
0 & I
\end{array}\right]\left[\begin{array}{ccc}
A & B_{1} & B_{2} \\
C_{2} & D_{21} & D_{22}
\end{array}\right]
$$

It is easily verified that (1) holds if and only if

$$
\left[\begin{array}{cc}
I & 0 \\
\Phi_{1, \Delta} & \Phi_{2, \Delta} \\
0 & I
\end{array}\right]^{T} L\left[\begin{array}{cc}
I & 0 \\
\Phi_{1, \Delta} & \Phi_{2, \Delta} \\
0 & I
\end{array}\right] \succ 0, \quad \forall \Delta \in \underline{\Delta}_{S}
$$

Letting $v_{1}$ and $v_{2}$ be appropriately sized vectors and defining $\xi_{\Delta}:=\Phi_{1, \Delta} v_{1}+\Phi_{2, \Delta} v_{2}$, the previous condition holds if and only if

$$
\left[\begin{array}{l}
v_{1} \\
\xi_{\Delta} \\
v_{2}
\end{array}\right]^{T} L\left[\begin{array}{l}
v_{1} \\
\xi_{\Delta} \\
v_{2}
\end{array}\right]>0, \quad \forall \Delta \in \underline{\Delta}_{s},\left[\begin{array}{l}
v_{1} \\
v_{2}
\end{array}\right] \neq 0
$$

We now characterize $\underline{\Delta}_{s}$. Note that

$$
\xi_{\Delta}=\left(I-\Delta D_{11}\right)^{-1} \Delta\left[\begin{array}{c}
C_{1}^{T} \\
D_{12}^{T}
\end{array}\right]^{T}\left[\begin{array}{l}
v_{1} \\
v_{2}
\end{array}\right] \Rightarrow \xi_{\Delta}=\Delta\left[\begin{array}{c}
C_{1}^{T} \\
D_{11}^{T} \\
D_{12}^{T}
\end{array}\right]^{T}\left[\begin{array}{l}
v_{1} \\
\xi_{\Delta} \\
v_{2}
\end{array}\right]
$$

Thus, $\xi_{\Delta}$ corresponds to a value of $\Delta \in \underline{\Delta}_{s}$ if and only if

$$
\xi_{\Delta}^{T} \xi_{\Delta} \leq\left[\begin{array}{c}
v_{1} \\
\xi_{\Delta} \\
v_{2}
\end{array}\right]^{T}\left[\begin{array}{c}
C_{1}^{T} \\
D_{11}^{T} \\
D_{12}^{T}
\end{array}\right]\left[\begin{array}{c}
C_{1}^{T} \\
D_{11}^{T} \\
D_{12}^{T}
\end{array}\right]^{T}\left[\begin{array}{l}
v_{1} \\
\xi_{\Delta} \\
v_{2}
\end{array}\right]
$$

With that characterization of $\underline{\Delta}_{S}$ in place, we now use the $\mathcal{S}$ procedure (see, e.g. [6]) to say that (2) holds if and only if $\exists \tau>0$ such that

$$
\begin{aligned}
& {\left[\begin{array}{l}
v_{1} \\
\xi_{\Delta} \\
v_{2}
\end{array}\right]^{T} L\left[\begin{array}{l}
v_{1} \\
\xi_{\Delta} \\
v_{2}
\end{array}\right]>\tau\left(\left[\begin{array}{l}
v_{1} \\
\xi_{\Delta} \\
v_{2}
\end{array}\right]^{T}\left[\begin{array}{c}
C_{1}^{T} \\
D_{11}^{T} \\
D_{12}^{T}
\end{array}\right]\left[\begin{array}{c}
C_{1}^{T} \\
D_{11}^{T} \\
D_{12}^{T}
\end{array}\right]^{T}\left[\begin{array}{l}
v_{1} \\
\xi_{\Delta} \\
v_{2}
\end{array}\right]-\xi_{\Delta}^{T} \xi_{\Delta}\right)} \\
& \forall\left[\begin{array}{lll}
v_{1}^{T} & \xi_{\Delta}^{T} & v_{2}^{T}
\end{array}\right] \neq 0 .
\end{aligned}
$$

With a little algebra, it can be shown that this is equivalent to $\mathcal{M}(\tau, P, W, V) \succ 0$. 
The previous theorem only considered the case when $\Delta \in \underline{\Delta}_{s}$. However, the next theorem tells us that if $\tau>0, P \succ 0$, and $\mathcal{M}(\tau, P, W, V) \succ 0$, we can conclude a much stronger resultrobust performance is guaranteed over all $\Delta(z) \in \underline{\Delta}_{d}$.

Theorem 3.4. If $\tau>0, P \succ 0$, and $\mathcal{M}(\tau, P, W, V) \succ 0$, then $\|G(z)\|_{2}^{2}<\operatorname{tr}\{W\}, \forall \Delta(z) \in \underline{\Delta}_{d}$.

Proof. Fix $\Delta(z) \in \Delta_{d}$. We now define $x_{i, 0}:=0, w_{i, 0}:=e_{i}, w_{i, k}:=$ $0, \forall k \in \mathbb{Z}_{>0}, i=1, \ldots, n_{d}$, where $e_{i}$ is the $i^{t h}$ standard Cartesian basis vector. Recall that the $\mathcal{H}_{2}$ norm can be interpreted in the time domain as

$$
\begin{gathered}
\|G(z)\|_{2}^{2}=\sum_{i=1}^{n_{d}} \sum_{k=0}^{\infty}\left[p_{i, k}^{T} p_{i, k}\right] \\
{\left[\begin{array}{c}
\frac{x_{i, k+1}}{q_{i, k}} \\
p_{i, k}
\end{array}\right]=\left[\begin{array}{c|cc}
A & B_{1} & B_{2} \\
\hline C_{1} & D_{11} & D_{12} \\
C_{2} & D_{21} & D_{22}
\end{array}\right]\left[\begin{array}{c}
x_{i, k} \\
d_{i, k} \\
w_{i, k}
\end{array}\right], \quad d_{i}(z)=\Delta(z) q_{i}(z) .}
\end{gathered}
$$

Since $\mathcal{M}(\tau, P, W, V) \succ 0$, we see that

$$
\left[\begin{array}{l}
x_{i, k} \\
d_{i, k} \\
w_{i, k}
\end{array}\right]^{T}\left[\begin{array}{lll}
P & 0 & V \\
\bullet & \tau I & 0 \\
\bullet & \bullet & W
\end{array}\right]\left[\begin{array}{l}
x_{i, k} \\
d_{i, k} \\
w_{i, k}
\end{array}\right] \geq\left[\begin{array}{c}
x_{i, k+1} \\
q_{i, k} \\
p_{i, k}
\end{array}\right]^{T}\left[\begin{array}{ccc}
P & 0 & 0 \\
0 & \tau I & 0 \\
0 & 0 & I
\end{array}\right]\left[\begin{array}{c}
x_{i, k+1} \\
q_{i, k} \\
p_{i, k}
\end{array}\right]
$$

where we have used the equations governing $G(z)$. Summing both sides from $k=0$ to $N$ yields, after a bit of simplification,

$$
e_{i}^{T} W e_{i} \geq x_{i, N+1}^{T} P x_{i, N+1}+\sum_{k=0}^{N}\left[p_{i, k}^{T} p_{i, k}+\tau\left(q_{i, k}^{T} q_{i, k}-d_{i, k}^{T} d_{i, k}\right)\right]
$$

Since $\|\Delta(z)\|_{\infty} \leq 1$ and $\Delta(z)$ is causal, we see that $\sum_{k=0}^{N}\left[q_{i, k}^{T} q_{i, k}-\right.$ $\left.d_{i, k}^{T} d_{i, k}\right] \geq 0$. Also, $P \succ 0 \Rightarrow x_{N+1}^{T} P x_{N+1} \geq 0$. Therefore,

$$
\begin{gathered}
e_{i}^{T} W e_{i} \geq \sum_{k=0}^{N}\left[p_{i, k}^{T} p_{i, k}\right], \quad \forall N \in \mathbb{Z}_{\geq 0} \\
\Rightarrow e_{i}^{T} W e_{i} \geq \sum_{k=0}^{\infty}\left[p_{i, k}^{T} p_{i, k}\right] \\
\Rightarrow \operatorname{tr}\{W\}=\sum_{i=1}^{n_{d}} e_{i}^{T} W e_{i} \geq \sum_{i=1}^{n_{d}} \sum_{k=0}^{\infty}\left[p_{i, k}^{T} p_{i, k}\right]=\|G(z)\|_{2}^{2}
\end{gathered}
$$

To recover the strict inequality, note that $\mathcal{M}(\tau, P, W-\varepsilon I, V) \succ$ 0 for sufficiently small $\varepsilon$. Thus, applying the above argument yields $\operatorname{tr}\{W-\varepsilon I\} \geq\|G(z)\|_{2}^{2} \Rightarrow \operatorname{tr}\{W\}>\|G(z)\|_{2}^{2}$. Since $\Delta(z)$ is arbitrary, this concludes the proof.

With this in place, a reasonable way to determine an upper bound on the robust $\mathcal{H}_{2}$ performance of $G(z)$ over all $\Delta(z) \in \underline{\Delta}_{d}$ is to solve the SDP

$$
\begin{aligned}
\min _{\tau, P, W, V} \operatorname{tr}\{W\} \\
\text { s.t. } \tau \geq 0, P \succeq 0, \mathcal{M}(\tau, P, W, V) \succeq 0
\end{aligned}
$$

using a solver such as SeDuMi [3] or the mincx function in the Robust Control Toolbox for MATLAB. Note that we have relaxed the strict inequalities to nonstrict inequalities. If the unrelaxed problem is feasible (i.e. if problem (3) is strictly feasible), then this is acceptable because any point that satisfies the constraints in (3) can be perturbed by an arbitrarily small amount to create a point that strictly satisfies those constraints. Thus, by the continuity of $\operatorname{tr}\{W\}$, we conclude that if $\tau, P, W, V$ satisfy the constraints in (3), then $\|G(z)\|_{2}^{2} \leq \operatorname{tr}\{W\}, \forall \Delta(z) \in \Delta_{d}$.

It is thus important to determine when (3) is strictly feasible. The following theorem establishes that robust stability over $\Delta_{d}$ is a necessary and sufficient condition for (3) to be strictly feasible.

Theorem 3.5. Define $\hat{\phi}:=\left(A, B_{1}, C_{1}^{T} C_{1}, D_{11}^{T} D_{11}-I, C_{1}^{T} D_{11}\right)$. The following are equivalent:

1. $\exists \tau>0, P \succ 0, W, V$ such that $\mathcal{M}(\tau, P, W, V) \succ 0$

2. $A$ is Schur and $\left\|\left[\begin{array}{c|c}A & B_{1} \\ \hline C_{1} & D_{11}\end{array}\right]\right\|_{\infty}<1$

3. A is Schur and the DARE $\mathcal{R}_{\hat{\phi}}(P)=0$ has a stabilizing solution $P_{0}$ such that $B_{1}^{T} P_{0} B_{1}+D_{11}^{T} D_{11}-I \prec 0$.

Proof. Since the equivalence of the last two conditions is standard in the literature, we only prove the equivalence of the first two conditions. Under the assumption that $A$ is Schur, note that for sufficiently small $\varepsilon>0$

$$
\begin{aligned}
& \left\|\left[\begin{array}{c|c}
A & B_{1} \\
\hline C_{1} & D_{11}
\end{array}\right]\right\|_{\infty}<1 \Leftrightarrow\left\|\left[\begin{array}{c|c}
A^{T} & C_{1}^{T} \\
\hline B_{1}^{T} & D_{11}^{T}
\end{array}\right]\right\|_{\infty}<1 \\
& \Leftrightarrow\left\|\left[\begin{array}{c|cc}
A^{T} & C_{1}^{T} & \varepsilon C_{2}^{T} \\
\hline B_{1}^{T} & D_{11}^{T} & \varepsilon D_{21}^{T}
\end{array}\right]\right\|_{\infty}<1 \Leftrightarrow\left\|\left[\begin{array}{c|cc}
A^{T} & \varepsilon^{-1} C_{1}^{T} & C_{2}^{T} \\
\hline B_{1}^{T} & \varepsilon^{-1} D_{11}^{T} D_{21}^{T}
\end{array}\right]\right\|_{\infty}^{2}<\varepsilon^{-2} .
\end{aligned}
$$

Defining $\tau:=\varepsilon^{-2}$, we use the LMI representation of the $\mathcal{H}_{\infty}$ norm (see, e.g. [7]) to see that this is equivalent to the existence of $P \succ 0$ (for sufficiently large $\tau>0$ ) such that

$$
\begin{aligned}
0 \prec\left[\begin{array}{cc}
P & 0 \\
0 & \tau I
\end{array}\right]-\left[\begin{array}{cc}
A & B_{1} \\
\sqrt{\tau} C_{1} & \sqrt{\tau} D_{11} \\
C_{2} & D_{21}
\end{array}\right]^{T}\left[\begin{array}{lll}
P & 0 & 0 \\
0 & I & 0 \\
0 & 0 & I
\end{array}\right]\left[\begin{array}{cc}
A & B_{1} \\
\sqrt{\tau} C_{1} & \sqrt{\tau} D_{11} \\
C_{2} & D_{21}
\end{array}\right] \\
=\left[\begin{array}{cc}
P & 0 \\
0 & \tau I
\end{array}\right]-\left[\begin{array}{cc}
A & B_{1} \\
C_{1} & D_{11} \\
C_{2} & D_{21}
\end{array}\right]^{T}\left[\begin{array}{ccc}
P & 0 & 0 \\
0 & \tau I & 0 \\
0 & 0 & I
\end{array}\right]\left[\begin{array}{cc}
A & B_{1} \\
C_{1} & D_{11} \\
C_{2} & D_{21}
\end{array}\right] .
\end{aligned}
$$

Since this matrix inequality is the first two rows and columns of the condition $\mathcal{M}(\tau, P, W, V) \succ 0$, applying Proposition 3.1 concludes the proof. 
Thus, we can find an upper bound on the robust $\mathcal{H}_{2}$ performance of $G(z)$ over all $\Delta(z) \in \underline{\Delta}_{d}$ by verifying condition 3 in Theorem 3.5 and then solving (3).

\section{DARE APPROACH TO H2 GUARANTEED COST ANALYSIS}

In the previous section, we demonstrated how an upper bound on robust $\mathcal{H}_{2}$ performance of $G(z)$ over all $\Delta(z) \in \Delta_{d}$ could be determined using convex optimization. In this section, we use the results of section 2 to show that the optimization problem can be solved using DAREs with parameters

$$
\begin{array}{ll}
B:=B_{1}, & Q:=\tau C_{1}^{T} C_{1}+C_{2}^{T} C_{2}, \\
R:=\tau\left(D_{11}^{T} D_{11}-I\right)+D_{21}^{T} D_{21}, & S:=\tau C_{1}^{T} D_{11}+C_{2}^{T} D_{21} .
\end{array}
$$

We first examine the strict feasibility of (3) for a fixed value of $\tau$.

Theorem 4.1. Let $\tau>0$ be given. $\exists P \succ 0, W, V$ such that $\mathcal{M}(\tau, P, W, V) \succ 0$ if and only if $A$ is Schur and the DARE $\mathcal{R}_{\phi}(P)=0$ has a stabilizing solution $P_{0}$ such that $B^{T} P_{0} B+R \prec 0$. Moreover, when A is Schur, $\mathcal{M}(\tau, P, W, V) \succ 0 \Rightarrow P \succ P_{0} \succeq 0$.

Proof. $(\Rightarrow)$ Let $\mathcal{M}(\tau, P, W, V) \succ 0, P \succ 0$. Looking at the upper left block of this inequality, we see that $P-A^{T} P A \succ Q \succeq 0$ which implies that $A$ is Schur. Note in particular that $(A, B)$ is trivially d-stabilizable. Removing the third row and column of $\mathcal{M}(\tau, P, W, V) \succ 0$, we see that $\mathcal{L}_{\phi}(P) \prec 0$. By Theorem 2.4, the DARE $\mathcal{R}_{\phi}(\bar{P})=0$ has a stabilizing solution $P_{0}$ such that $B^{T} P_{0} B+R \prec 0$. By Corollary 2.6, this means that $P \succ P_{0}$. We now note that the dare can be written

$$
\begin{aligned}
P_{0}-A^{T} P_{0} A & =Q-\left(A^{T} P_{0} B+S\right)\left(B^{T} P_{0} B+R\right)^{-1}\left(B^{T} P_{0} A+S^{T}\right) \\
& \succeq Q \succeq 0 .
\end{aligned}
$$

Since $A$ is Schur, this implies that $P_{0} \succeq 0$.

$(\Leftarrow)$ Let $A$ be Schur and the DARE have a stabilizing solution such that $B^{T} P_{0} B+R \prec 0$. Since $(A, B)$ is trivially dstabilizable, we apply Theorem 2.4 to conclude that $\exists P$ such that $\mathcal{L}_{\phi}(P) \prec 0$. The upper left block of this inequality tells us that $P-A^{T} P A \succ Q \succeq 0$. Since $A$ is Schur, this implies that $P \succ 0$. Noting that $\mathcal{L}_{\phi}(P) \prec 0$ is the first two rows and columns of the condition $\mathcal{M}(\tau, P, W, V) \succ 0$, we apply Proposition 3.1 to finish the proof.

The following Theorem establishes that, for a fixed value of $\tau$, the optimization over the remaining parameters to find the best upper bound of $\mathcal{H}_{2}$ guaranteed cost performance can be solved using a single DARE.

Theorem 4.2. Let $\tau>0$ be given and assume that $\exists P \succ 0, W, V$ such that $\mathcal{M}(\tau, P, W, V) \succ 0$. Let $P_{0}$ be the stabilizing solution of the DARE $\mathcal{R}_{\phi}(P)=0$ and define

$$
\begin{aligned}
S_{13} & :=A^{T} P_{0} B_{2}+\tau C_{1}^{T} D_{12}+C_{2}^{T} D_{22} \\
S_{23} & :=B^{T} P_{0} B_{2}+\tau D_{11}^{T} D_{12}+D_{21}^{T} D_{22} \\
S_{33} & :=B_{2}^{T} P_{0} B_{2}+\tau D_{12}^{T} D_{12}+D_{22}^{T} D_{22} \\
V_{0} & :=S_{13}-\left(A^{T} P_{0} B+S\right)\left(B^{T} P_{0} B+R\right)^{-1} S_{23} \\
W_{0} & :=S_{33}-S_{23}^{T}\left(B^{T} P_{0} B+R\right)^{-1} S_{23} .
\end{aligned}
$$

Then

$$
\begin{aligned}
\operatorname{tr}\left\{W_{0}\right\}=\min _{P, W, V} \operatorname{tr}\{W\} \\
\text { s.t. } P \succeq 0, \mathcal{M}(\tau, P, W, V) \succeq 0
\end{aligned}
$$

and an optimizer is given by $(P, W, V)=\left(P_{0}, W_{0}, V_{0}\right)$.

Proof. First note that the existence of $P_{0}$ is guaranteed by Theorem 4.1 and satisfies $P_{0} \succeq 0$. We begin by proving that the claimed optimizer is feasible. Note that

$$
\mathcal{M}\left(\tau, P_{0}, W_{0}, V_{0}\right)=\left[\begin{array}{ccc}
P_{0}-A^{T} P_{0} A-Q & -A^{T} P_{0} B-S & V_{0}-S_{13} \\
\bullet & -B^{T} P_{0} B-R & -S_{23} \\
\bullet & \bullet & W_{0}-S_{33}
\end{array}\right]
$$

Since symmetrically permuting the rows and columns of a symmetric matrix do not affect the positive semi-definiteness of the matrix, we exchange the second and third rows and columns and note that the Schur complement of $-\left(B^{T} P_{0} B+R\right)$ is 0 . Since $-\left(B^{T} P_{0} B+R\right) \succ 0$ and its Schur complement is positive semidefinite, this implies that $\mathcal{M}\left(\tau, P_{0}, W_{0}, V_{0}\right) \succeq 0$. Thus, the claimed optimizer is feasible.

Now we show that it is, indeed, optimal. Let $\bar{P} \succ 0, \bar{W}, \bar{V}$ satisfy $\mathcal{M}(\tau, \bar{P}, \bar{W}, \bar{V}) \succ 0$. By Theorem $4.1, \bar{P} \succ P_{0}$. Eliminating the first row and column of $\mathcal{M}(\tau, \bar{P}, \bar{W}, \bar{V})$, we see that

$$
\begin{aligned}
0 & \prec\left[\begin{array}{cc}
\tau I & 0 \\
0 & \bar{W}
\end{array}\right]-\left[\begin{array}{cc}
B_{1} & B_{2} \\
D_{11} & D_{12} \\
D_{21} & D_{22}
\end{array}\right]^{T}\left[\begin{array}{ccc}
\bar{P} & 0 & 0 \\
0 & \tau I & 0 \\
0 & 0 & I
\end{array}\right]\left[\begin{array}{cc}
B_{1} & B_{2} \\
D_{11} & D_{12} \\
D_{21} & D_{22}
\end{array}\right] \\
& \preceq\left[\begin{array}{cc}
\tau I & 0 \\
0 & \bar{W}
\end{array}\right]-\left[\begin{array}{cc}
B_{1} & B_{2} \\
D_{11} & D_{12} \\
D_{21} & D_{22}
\end{array}\right]^{T}\left[\begin{array}{ccc}
P_{0} & 0 & 0 \\
0 & \tau I & 0 \\
0 & 0 & I
\end{array}\right]\left[\begin{array}{cc}
B_{1} & B_{2} \\
D_{11} & D_{12} \\
D_{21} & D_{22}
\end{array}\right] \\
& =\left[\begin{array}{cc}
-B^{T} P_{0} B-R & -S_{23} \\
\bullet & \bar{W}-S_{33}
\end{array}\right]
\end{aligned}
$$

By Schur complements, this implies that $\bar{W} \succ W_{0}$, which implies that $\operatorname{tr}\{\bar{W}\}>\operatorname{tr}\left\{W_{0}\right\}$. Now let $P \succeq 0, W, V$ satisfy $\mathcal{M}(\tau, P, W, V) \succeq 0$ and define $P_{\lambda}:=\lambda \bar{P}+(1-\bar{\lambda}) P, W_{\lambda}:=\lambda \bar{W}+$ 
$(1-\lambda) W$, and $V_{\lambda}:=\lambda \bar{V}+(1-\lambda) V$. Note that $P_{\lambda} \succ 0, \forall \lambda \in(0,1]$ and

$$
\begin{aligned}
\mathcal{M}\left(\tau, P_{\lambda}, W_{\lambda}, V_{\lambda}\right) & =\lambda \mathcal{M}(\tau, \bar{P}, \bar{W}, \bar{V})+(1-\lambda) \mathcal{M}(\tau, P, W, V) \\
& \succ 0, \quad \forall \lambda \in(0,1] .
\end{aligned}
$$

By the same argument as was used to prove that $\bar{W} \succ W_{0}$, we see that $W_{\lambda} \succ W_{0}, \forall \lambda \in(0,1]$. Since $W_{\lambda}$ is continuous in $\lambda$, we conclude that $W_{\lambda} \succeq W_{0}$ when $\lambda=0$, i.e. $W \succeq W_{0}$. This in turn implies that $\operatorname{tr}\{W\} \geq \operatorname{tr}\left\{W_{0}\right\}$.

Note that we can reformulate (3) as the nested optimization

$$
\min _{\tau \geq 0}\left(\begin{array}{l}
\min _{P, W, V} \operatorname{tr}\{W\} \\
\text { s.t. } P \succeq 0, \mathcal{M}(\tau, P, W, V) \succeq 0
\end{array}\right) .
$$

By the properties of convex optimization, the outer optimization is a nonlinear convex optimization [8] and, by the preceding theorem, the inner optimization can be performed using a single DARE. Now we present a result which makes it especially easy to find feasible values of $\tau$.

Proposition 4.3. Let $\bar{\tau}>0$ be given. If $\exists P \succ 0, W, V$ such that $\mathcal{M}(\bar{\tau}, P, W, V) \succ 0$, then $\forall \tau \geq \bar{\tau}, \exists P \succ 0, W, V$ such that $\mathcal{M}(\tau, P, W, V) \succ 0$.

Proof. Let $\mathcal{M}(\bar{\tau}, P, W, V) \succ 0$ and choose $\alpha \geq 1$. Note that

$$
\begin{aligned}
{\left[\begin{array}{ccc}
\alpha P & 0 & \alpha V \\
\bullet & \alpha \bar{\tau} I & 0 \\
\bullet & \bullet & \alpha W
\end{array}\right] } & \succ\left[\begin{array}{ccc}
A & B_{1} & B_{2} \\
C_{1} & D_{11} & D_{12} \\
C_{2} & D_{21} & D_{22}
\end{array}\right]^{T}\left[\begin{array}{ccc}
\alpha P & 0 & 0 \\
0 & \alpha \bar{\tau} I & 0 \\
0 & 0 & \alpha I
\end{array}\right]\left[\begin{array}{ccc}
A & B_{1} & B_{2} \\
C_{1} & D_{11} & D_{12} \\
C_{2} & D_{21} & D_{22}
\end{array}\right] \\
& \succeq\left[\begin{array}{ccc}
A & B_{1} & B_{2} \\
C_{1} & D_{11} & D_{12} \\
C_{2} & D_{21} & D_{22}
\end{array}\right]^{T}\left[\begin{array}{ccc}
\alpha P & 0 & 0 \\
0 & \alpha \bar{\tau} I & 0 \\
0 & 0 & I
\end{array}\right]\left[\begin{array}{ccc}
A & B_{1} & B_{2} \\
C_{1} & D_{11} & D_{12} \\
C_{2} & D_{21} & D_{22}
\end{array}\right] .
\end{aligned}
$$

Thus, $\mathcal{M}(\alpha \bar{\tau}, \alpha P, \alpha W, \alpha V) \succ 0, \forall \alpha \geq 1$.

We now analyze how $\operatorname{tr}\left\{W_{0}\right\}$ (as defined in Theorem 4.2) varies as $\tau$ is varied. Since the stabilizing solution of a DARE is analytic at any point at which it exists [9], $P_{0}$ (and, hence, $\operatorname{tr}\left\{W_{0}\right\}$ ) is an infinitely differentiable function of $\tau$ at any value of $\tau$ for which the DARE admits a stabilizing solution. In particular, by implicitly differentiating the DARE $\mathcal{R}_{\phi}(P)=0$, it can be shown that the derivative of $P_{0}$ with respect to $\tau$ satisfies the discrete Lyapunov equation

$$
\begin{aligned}
P_{0}^{\prime}=\mathcal{A}_{\phi}\left(P_{0}\right)^{T} P_{0}^{\prime} \mathcal{A}_{\phi}\left(P_{0}\right)-\mathcal{K}_{\phi}\left(P_{0}\right)^{T} \mathcal{K}_{\phi}\left(P_{0}\right) \\
+\left(C_{1}+D_{11} \mathcal{K}_{\phi}\left(P_{0}\right)\right)^{T}\left(C_{1}+D_{11} \mathcal{K}_{\phi}\left(P_{0}\right)\right) .
\end{aligned}
$$

Since $\mathcal{A}_{\phi}\left(P_{0}\right)$ is Schur, this Lyapunov function can always be uniquely solved for $P_{0}^{\prime}$. We can then compute the derivative of

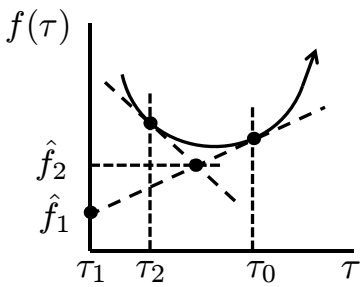

Figure 2. ILLUSTRATION OF LOWER BOUND COMPUTATION

the upper bound on the $\mathcal{H}_{2}$ guaranteed cost performance with respect to $\tau$ as

$$
\begin{aligned}
\operatorname{tr}\left\{W_{0}\right\}^{\prime}= & \operatorname{tr}\left\{\left(B_{2}+B_{1} K_{23}\right)^{T} P_{0}^{\prime}\left(B_{2}+B_{1} K_{23}\right)\right. \\
& \left.+\left(D_{12}+D_{11} K_{23}\right)^{T}\left(D_{12}+D_{11} K_{23}\right)-K_{23}^{T} K_{23}\right\} \\
K_{23}:= & -\left(B^{T} P_{0} B+R\right)^{-1} S_{23} .
\end{aligned}
$$

Since (4) is a convex optimization, $\operatorname{tr}\left\{W_{0}\right\}^{\prime}$ is a monotonic nondecreasing function of $\tau$. Also, the condition $\operatorname{tr}\left\{W_{0}\right\}^{\prime}=0$ is sufficient to guarantee optimality.

The value and derivative of $\operatorname{tr}\left\{W_{0}\right\}$ is also useful for generating a lower bound on the optimal value of (4). Consider Figure 2 , which shows a graph of a convex function $f(\tau)$ in which $\tau_{0}$ is known to be an upper bound on the minimizing value of $\tau$. By convexity, if $\tau_{1}$ is known to be a lower bound on the minimizing value of $\tau$, the value and derivative of $f$ at $\tau_{0}$ gives us the lower bound $\hat{f}_{1}$. If instead, the value and derivative of $f$ at $\tau_{0}$ and $\tau_{2}$ is known, we have the lower bound $\hat{f}_{2}$. It should be noted that the second of these lower bounds is less conservative when it is applicable.

With these results in place, we can easily solve (4) using the following methodology:

Step 1-Check Feasibility: Use condition 3 of Theorem 3.5 to determine whether or not (4) is strictly feasible.

Step 2-Find Initial Interval: Choose $\alpha>1$. By Proposition 4.3, the DARE $\mathcal{R}_{\phi}(P)=0$ will have a stabilizing solution with the required properties for $\tau=\alpha^{k}$, for large enough $k$. Starting from $k=0$, iterate over $k$ until a value of $\tau=\alpha^{k}$ is found such that the DARE has a stabilizing solution $P_{0}$ that satisfies $B^{T} P_{0} B+R \prec 0$ and $\operatorname{tr}\left\{W_{0}\right\}^{\prime}>0$. Denote this value of value of $\tau$ by $\tau_{u}$. Note that this corresponds to an upper bound on the optimal value of $\tau$. If $\tau_{u}=1$, then 0 is a lower bound on the optimal value of $\tau$, otherwise $\tau_{u} / \alpha$ is a lower bound.

Step 3-Bisection: Solve the equation $\operatorname{tr}\left\{W_{0}\right\}^{\prime}=0$ over $\tau$ using bisection. Whenever the DARE $\mathcal{R}_{\phi}(P)=0$ does not have a solution with the required properties for a given value of $\tau$, this corresponds to a lower bound on the optimal value of $\tau$.

In our implementation, we use $\alpha=100$. Also, except when the lower bound on the optimal value of $\tau$ is 0 , we use the geometric 


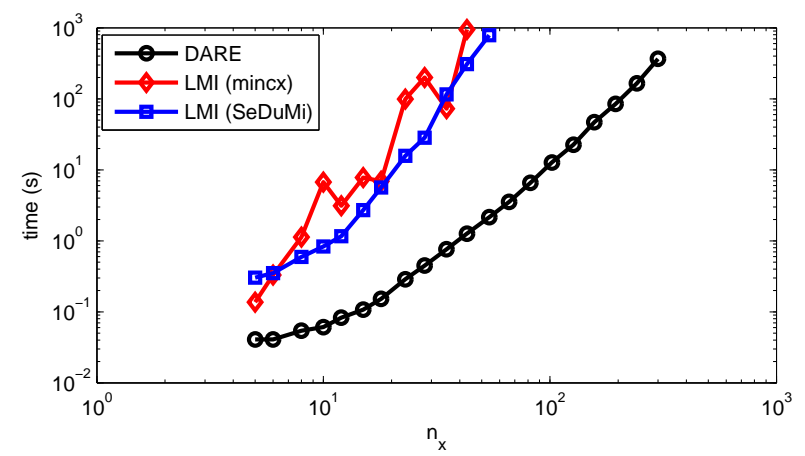

Figure 3. TIME REQUIRED TO SOLVE RANDOMLY GENERATED H2 GUARANTEED COST PERFORMANCE ANALYSIS PROBLEMS

mean instead of the arithmetic mean to better deal with large intervals in which the optimal value of $\tau$ could lie. In the bisection step, we use two stopping criteria; if we define the relative error as $v:=1-f / \operatorname{tr}\left\{W_{0}\right\}$ where $f$ is the lower bound computed as shown in Figure 2, we terminate the algorithm when either $v<10^{-10}$ or the number of iterations (including the iterations required to find the initial interval) exceeds 30 .

\section{NUMERICAL EXPERIMENTS}

In this section, we consider the application of the developed methodologies to randomly generated $\mathcal{H}_{2}$ guaranteed cost performance problems. In particular, we consider three approachesusing the DARE approach outlined in section 4, solving (3) using SeDuMi (parsed using YALMIP [10]), and solving (3) using the mincx command in the Robust Control Toolbox. The last two of these methods will be collectively called the LMI methods. It should be noted that YALMIP was not used when using mincx because YALMIP causes mincx to run more slowly. All numerical experiments were performed in MATLAB (with multithreaded computation disabled) on a computer with a $2.2 \mathrm{GHz}$ Intel Core 2 Duo Processor and 2 GB of RAM.

To generate the random systems in our numerical experiments, we first generated a random stable discrete-time state space system using drss in MATLAB and then multiplied the system by the inverse of its $\mathcal{H}_{\infty}$ norm (computed by norm). This system was then multiplied by a random number generated from a uniform distribution on $[-1,1]$. The resulting system corresponded to generating random values of $A, B_{1}, C_{1}$, and $D_{11}$ for a robustly stable system. The entries of $B_{2}, C_{2}, D_{12}, D_{21}$, and $D_{22}$ were generated randomly from independent normal distributions. For all of the numerical experiments, we chose the signal dimensions to be $n_{q}=5, n_{d}=6, n_{p}=7, n_{w}=8$.

In the first experiment, we tested the speed of the methodologies over several values of $n_{x}$. The results of this test are shown in Figure 3. In particular, note that the DARE method is faster than the LMI methods for all of the randomly generated problems. For instance, for the $43^{\text {rd }}$-order system, it respectively took the DARE approach, the mincx approach, and

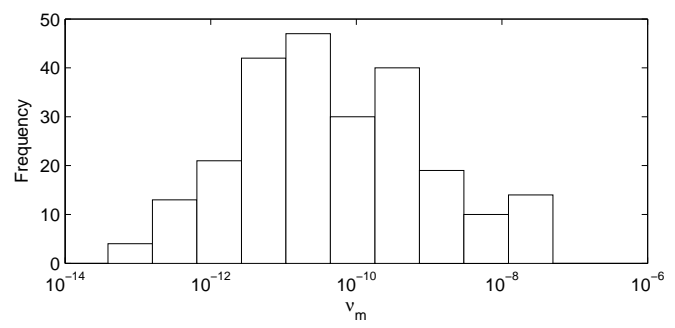

Figure 4. HISTOGRAM OF $v_{m}$ WHEN MINCX REPORTS SMALLER COST THAN DARE APPROACH

the SeDuMi approach 1.27 seconds (averaged over several runs), 947.75 seconds, and 307.76 seconds to compute the upper bound on the $\mathcal{H}_{2}$ guaranteed cost performance. Also note that the DARE method appears to have a complexity of $O\left(n_{x}^{3}\right)$ whereas the SeDumi method appears to have a complexity of $O\left(n_{x}^{5}\right)$. The curve which corresponds to the mincx method is not smooth because the number of iterations required to solve the problem often changes dramatically from problem to problem, unlike the other two methods. Nonetheless, since the computational time required for the mincx method is similar to that required by the SeDuMi method, it appears to have a complexity of roughly $O\left(n_{x}^{5}\right)$ also. Thus, the difference in computational speed between the DARE approach and the other two approaches becomes more pronounced for larger values of $n_{x}$.

In the second experiment, we tested the accuracy of the DARE approach compared to the LMI approaches for 400 randomly generated analysis problems with $n_{x}=20$. To this end, we first define $f_{d}, f_{m}$, and $f_{s}$ as the upper bounds on the $\mathcal{H}_{2}$ guaranteed cost performance for a given system respectively computed using the DARE approach, mincx, and SeDuMi. The criterion we will be using to compare the accuracy of the relevant methods is the relative error, i.e. we use the criterion $v_{m}:=\left|1-f_{m} / f_{d}\right|$ to compare the accuracy of the mincx approach to the DARE approach and the criterion $v_{s}:=\left|1-f_{s} / f_{d}\right|$ to compare the accuracy of the SeDuMi approach to the DARE approach. For both comparisons, the results are split into two categories - cases in which the LMI approach reports a smaller cost and cases in which the DARE approach reports a smaller cost.

Figure 4 shows the histogram of $v_{m}$ for the cases in which mincx reports a smaller cost than the DARE approach. Note that all of these values are small, i.e. the mincx approach never significantly beats the DARE approach in terms of accuracy. Figure 5 shows the histogram of $v_{m}$ for the cases in which mincx reports a larger cost than the DARE approach. This figure shows that the DARE approach often report a much smaller cost than the mincx approach. Since both of these methods require all of the iterates to be feasible, we conclude that the DARE approach had better accuracy in these cases and the difference in reported cost is due to mincx getting "stuck" due to numerical problems. Figure 6 shows the histogram of $v_{s}$ for the cases in which SeDuMi reports a smaller cost than the DARE approach. Unlike the mincx approach, the SeDuMi approach generates 


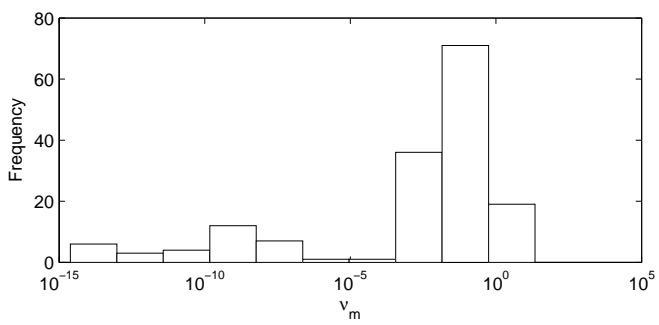

Figure 5. HISTOGRAM OF $v_{m}$ WHEN MINCX REPORTS LARGER COST THAN DARE APPROACH

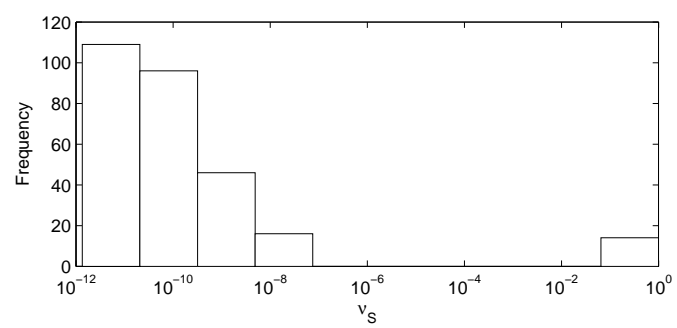

Figure 6. HISTOGRAM OF $v_{S}$ WHEN SEDUMI REPORTS SMALLER COST THAN DARE APPROACH

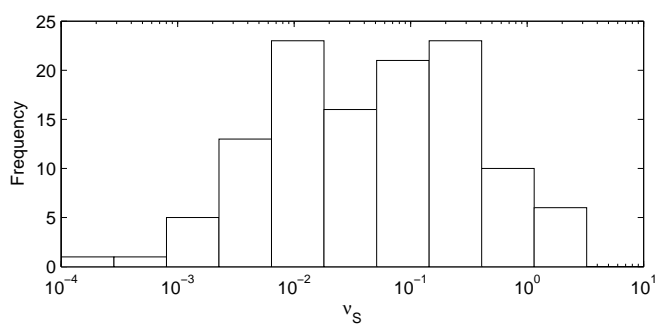

Figure 7. HISTOGRAM OF $v_{S}$ WHEN SEDUMI REPORTS LARGER COST THAN DARE APPROACH

significantly smaller costs than the DARE approach for 14 cases. However, for each of these 14 cases, the cost of the nominal system computed by the norm function in MATLAB is larger than the $\mathcal{H}_{2}$ guaranteed cost determined by SeDuMi. Since this is a contradiction, we conclude that these results correspond to cases in which SeDuMi failed. In particular, since SeDuMi uses infeasible path-following algorithms, not all of the iterates are guaranteed to be feasible. In these 14 cases, the final iterate was not feasible. Since, in infinite precision, the feasibility of one iterate implies feasibility of the next iterate for these infeasible pathfollowing algorithms, it is likely that SeDuMi failed to find any feasible iterates for these 14 cases. Figure 7 shows the histogram of $v_{s}$ for the cases in which SeDuMi reports a larger cost than the DARE approach. This figure shows that, like the mincx method, the SeDuMi method sometimes gets "stuck" due to numerical problems. Based on this, we conclude that the accuracy of the DARE method is generally superior to that of the LMI methods.

\section{CONCLUSION}

In this paper, we formulated the problem of finding the best upper bound on $\mathcal{H}_{2}$ guaranteed cost performance of a discretetime system with dynamic norm-bounded unstructured uncertainty as a SDP. We then demonstrated that exploiting the structure of this optimization by using the solution of DAREs increases the speed and accuracy with which we can solve these problems.

\section{ACKNOWLEDGMENT}

This work was supported in part by National Science Foundation grant CMS-0428917, the Information Storage Industry Consortium and the Computer Mechanics Laboratory at UC Berkeley.

\section{REFERENCES}

[1] Paganini, F., 1999. "Frequency domain conditions for robust $\mathcal{H}_{2}$ performance". IEEE Transactions on Automatic Control, 44(1), January, pp. 38-49.

[2] Petersen, I. R., McFarlane, D. C., and Rotea, M. A., 1998. "Optimal guaranteed cost control of discrete-time uncertain linear systems". International Journal of Robust and Nonlinear Control, 8(8), July, pp. 649-657.

[3] Sturm, J. F., 1999. "Using SeDuMi 1.02, a MATLAB toolbox for optimization over symmetric cones". Optimization Methods and Software, 11, pp. 625-653.

[4] Stoorvogel, A. A., 1993. "The robust $H_{2}$ control problem: A worst-case design". IEEE Transactions on Automatic Control, 38(9), September, pp. 1358-1370.

[5] Lancaster, P., and Rodman, L., 1995. Algebraic Riccati Equations. Oxford Science Publications. Clarendon Press, Oxford.

[6] Boyd, S., El Ghaoui, L., Feron, E., and Balakrishnan, V., 1994. Linear Matrix Inequalities in System and Control Theory, Vol. 15 of SIAM Studies in Applied Mathematics. Society for Industrial and Applied Mathematics, Philadelphia.

[7] de Oliveira, M. C., Geromel, J. C., and Bernussou, J., 2002. "Extended $H_{2}$ and $H_{\infty}$ norm characterizations and controller parametrizations for discrete-time systems". International Journal of Control, 75(9), June, pp. 666-679.

[8] Boyd, S., and Vandenberghe, L., 2004. Convex Optimization. Cambridge University Press, Cambridge, UK.

[9] Delchamps, D. F., 1983. "Analytic stabilization and the algebraic Riccati equation". In The 22nd IEEE Conference on Decision and Control, Vol. 22, pp. 1396-1401.

[10] Löfberg, J., 2004. "YALMIP : A toolbox for modeling and optimization in MATLAB". In Proceedings of the CACSD Conference. 